\title{
Study on the Collaborative Development of Beijing and Tianjin and Hebei Province from the Perspective of Low Carbon Economy
}

\author{
Qiong Luo \\ Institute of Economic Development Strategy \\ Party School of Tianjin Committee of C.P.C. \\ Tianjin, China, 300191
}

\begin{abstract}
Nowadays, the Beijing-Tianjin-Hebei region has become one of the three important poles of the economic growth in China, and the collaborative development of Beijing, Tianjin and Hebei province has been upgraded to a national strategy. However, during the collaborative development of Beijing, Tianjin and Hebei Province, there exist such problems as extensive development mode, uncoordinated development and serious environmental damages. The Beijing-Tianjin-Hebei region should catch the opportunities to speed up the adjustment of industrial structure, advance the low-carbon economy and establish collaborative cooperation in key areas on the basis of the strengths and weaknesses of their own.
\end{abstract}

Keywords-low-carbon economy; Beijing-Tianjin-Hebei collaborative development; low-carbon tourism; low-carbon agriculture

\section{INTRODUCTION}

Analyzing from overall situation across the country, the Beijing-Tianjin-Hebei region ranks ahead of others in regional economic development whether in population density, economic vitality, openness or in innovation ability. And the Beijing-Tianjin-Hebei region is a big engine to impel the economic development in China. Being such a critical area, it has instead become a place highlighting the most conflicts between population, environment and resources in East China.

The 2015 National Environmental Quality Report issued by Ministry of Environmental Protection shows that the Beijing-Tianjin-Hebei region has been in extremely serious air pollution across the country. And there were six cities in the Beijing-Tianjin-Hebei region, ranking within the last ten cities with poor air quality.

Bio-environmental protection has been taken as a key field for the first solution for the Beijing-Tianjin-Hebei collaborative development. As far as the bio-environmental protection, the three areas are required to break the administrative regional restrictions, promote the green cycle and low-carbon development and strengthen the bioenvironment protection and governance.

\section{FOUNDATIONS FOR THE COLLABORATIVE} DEVELOPMENT OF BEIJING, TIANJIN AND HEBEI PROVINCE

One of the foundations for the collaborative development of Beijing, Tianjin and Hebei Province is the difference in the conditions needed for the development of low-carbon economy. According to the theory of comparative advantage, even if the economic strength between two countries is in a wide difference, they, having comparative advantages and comparative disadvantages of their own, still keep trade cooperation available between them. The theory of comparative advantages is also applicable for Beijing, Tianjin and Hebei Province, despite the wide disparity between Hebei and Beijing and Tianjin, they still have advantages and disadvantages of their own, and it is completely possible for Beijing, Tianjin and Hebei to achieve collaborative development through the establishment of an effective cooperation mechanism.

\section{A. Advantages and Disadvantages Owned by Beijing}

1) Advantages: Beijing has entered the postindustrialization period, and its output value ratio of tertiary industry accounts for more than $70 \%$, showing a typical inverted pyramid structure. Through a lot of analysis, we have mastered that that the energy consumption intensity of the tertiary industry has been much lower than that of the secondary industry and the primary industry, which owns a favorable advantage in foundation for Beijing to achieve the energy-saving and emission reduction targets. In addition, there stand a large number of top universities and scientific research institutions leading at home, which have cultivated a large number of low-carbon scientific research talents, owning the most advanced energy-saving and emission reduction technologies at home. The advantages of human capital and science and technologies that Beijing owns can not be compared by Tianjin, Hebei and other provinces or cities at home.

2) Disadvantages: Beijing has bee in a weak bioenvironment, and the rapid economic development and sharp population expansion have caused the extreme shortage of water resources which are scarce basically, and the pollution of environment especially the air is becoming serious 
increasingly; the low urban vegetation coverage and its extreme reach to the sand source in the northwest has caused Beijing to be exposed to sandstorms. In addition, Beijing is very scarce in energy, which is far to meet the demand, and has to be introduced from the outside.

\section{B. Advantages and Disadvantages Owned by Tianjin}

1) Advantages: The adequate funding and preferential policy support in Binhai New Area have attracted the eyeballs of enterprises which are engaged in the production of environment friendly and low-carbon products, and many companies gathered here, Tianjin has been gradually in the leading of the field of low-carbon equipment manufacturing in the country.

In the past decades, Tianjin has its economic development fully supported by the central government both in finance and policies. Tianjin is orientated to be the economic center of the north, and the construction of Tianjin Binhai New Area has been upgraded to a national strategy, which aims to be build a new urban characterized of low carbon, recycle, green and livableness. Binhai New Area has attracted a large number of new energy enterprises and made great achievements in improving energy efficiency and developing new energy. In order to build the Binhai New Area into a leading low-carbon economy demonstration area across the country, the governmental authorities have invested a lot of manpower, materials and financial resources and formulated favorable policies to support the R\&D of low-carbon technologies and the introduction of scientific talents.

On other hand, Tianjin Climate Exchange was formally established in 2009, which is the first comprehensive environmental rights trading institution in China, and also a major financial innovation on the climate mechanism, and it has put a solid foundation for the development of low-carbon economy in Tianjin.

2) Disadvantages: First of all, Tianjin is in the middle and late stage of industrialization, heavy and chemical industries got rapid progress. With the continuous development and opening up of the Binhai New Area, some of large petrochemical heavy construction projects, which will bring pollution to the environment, started or launched, on the one hand, the large projects advance the economic growth of the Binhai New Area, on the other hand, it keeps the secondary industry output value higher in Tianjin. It is well known that energy consumption by unit output value of the secondary industry is much higher than that of the tertiary industry, so does the carbon emission in unit output value. If planning to develop the low-carbon economy, Tianjin has to accelerate the transformation of economic development pattern and achieve the optimization and upgrading of industrial structures.

Second, although there are many large and technologically advanced multinational companies stationed in Tianjin, ye the multinational companies disclose no key technologies to Chinese enterprises, and national enterprises are still incompetent in technical innovation and it is really not easy for medium and small enterprises at home to maintain basic development, the low-carbon technology is far to reach.

\section{Advantages and Disadvantages Owned by Hebei}

1) Advantages: Hebei has abundant energy resources, which is a prominent advantage, so it has great potentials in the development of energy industry. The solar energy in Baoding and Xingtai and the wind energy and geothermal energy in Zhangjiakou and Chengde are both clean energy with huge development potentials, which are inexhaustible to some degree.

The PV industry in Baoding and Xingtai has begun to take shape, based on which, a great number of famous enterprises, independent intellectual property rights, emerging in recent years may further promote the solar energy PV grid-connected power generation, build more light energy demonstration projects and drive the PV industry to the higher end. Zhangjiakou and Chengde are located at the upper entrance of Beijing and Tianjin, owning advantageous geographical location. The abundant wind energy and geothermal energy in the area can be made use of in the future to build a wind energy and geothermal energy industrial base. Moreover, the ecological environments in Zhangijiakou and Chengde are better, and the beautiful scenery makes it applicable for the development of low-carbon tourism.

In addition, Hebei is a large province in agriculture, which has a favorable basis for the development of low-carbon agriculture. For example, during the development of aquaculture, Handan succeeded in achieving the recycling use of emissions from livestock and poultry through the medium methane project relying on the farm, which not only bring material benefits to farmers but also protect the environment effectively; besides, the bio-agriculture development with the biogas residual and slurry highly used in Handan city has brought sound demonstration effect to the low carbon agriculture development at home.

2) Disadvantages: Hebei Province is in the middle stage of industrialization, leading industries as steel, petrochemical, building materials, coal mining, power and heat supply and so are all of high energy consumption and high pollution, causing higher carbon dioxide emission. The poor industrial development and lower proportion of tertiary industry in Hebei Province are major causes for the less cooperation between Hebei and Beijing and Tianjin. The rich natural resources, which should be a major advantage for Hebei Province, finally causes the economic growth of Hebei Province to seriously depend on the traditional high energy consumption industries, and unable to get it off, which is clearly contrary to the requirements of developing low-carbon economy. How to get rid of the dependence on coal and the restriction of traditional industries has been a major problem for Hebei to face.

The development of low-carbon economy can not be separated from the cultivation of low-carbon technical talents, Hebei Province, with a population of 70 million people, supposedly should not lack scientific research talents, however the poor economy and disqualified scientific research environment in Hebei Province have finally led the outflow of high-quality talents to large cities such as Beijing, Shanghai, Guangzhou and Shenzhen, as a result, Hebei is lost in the trouble difficult to attract and retain talents, making it extremely scarce in low-carbon human capital. In addition, the 
adjustment of industrial structure, cultivation of technical talents and the development of low-carbon economy is not as easy as spoken, and it has been supported by a lot of funds, which, for the undeveloped Hebei Province, is a heavy burden.

\section{PROMINENT PROBleMS EXISTING FOR THE BEIJING- TIANJIN-HEBEI COOPERATION}

\section{A. Cooperation Mechanism Poor in Low Carbon}

Although the low carbon industry as new energy in Beijing, Tianjin and Hebei has formed a certain foundation and scale, yet the mechanism to strengthen low-carbon cooperation is not perfect, lack in grouping for collaborative innovation, still lost in the traditional thought pattern "Self-owned lands" criticized by President Xi Jinping. For example, the products of PV industry in Baoding has less application in Beijing, though Beijing has good light intensity, yet the use of solar energy equipment in the field is much less. The service of technical talents in Beijing to Tianjin and Hebei is inadequate. The Beijing-Tianjin-Hebei region and convenient transportation make the low-carbon products and service broad in market space. However, due to the high costs of new energy power generation, especially optoelectronics with higher obstacles in the domestic application. In other policies and industrial fields, the cooperation mechanism between Beijing, Tianjin and Hebei is imperfect, for example, and there is equal public service in college entrance examination, primary education, social medical care, endowment and traffic facilities; there are obvious difference in policy service, infrastructure and public service, which cause siphonic effect and polar effect of Beijing and Tianjin to talents and capital, high inflow and less outflow of talents, as well as little spread and contribution to undeveloped area in Hebei.

\section{B. The Same Trends of Key Industries in Beijing, Tianjin and Hebei}

For a long time, the industrial overlap of key industrial in Beijing, Tianjin and Hebei has not been solved, which become serious gradually. The "Twelfth Five-Year Plans" in Beijing, Tianjin and Hebei show, most of the key industries in the three regions are similar: Beijing, Tianjin and Hebei all take new energy, equipment manufacturing and medicine as a key industry; Hebei and Tianjin have similar industrial layout in petrochemical and textile; Beijing and Tianjin both plan to develop the aviation and space industry, electronic information industry and automobile manufacturing industry; Beijing, Tianjin and Hebei have more similarities in the service industry, and all focus on the development of finance, culture, logistics and tourism, etc., Hebei and Tianjin have the industrial overlap in the service outsourcing.

\section{Poor Drive of Central Cities and Lack of Deep Cooperation}

Beijing, Tianjin and Hebei, acting as an important economic growth pole in the north, is some unworthy of the name, compared to Guangzhou in the Pearl River Delta and Shanghai in the Yangtze River Delta, Beijing and Tianjin make no effect as it should do, it not only fails to drive the development of Hebei Province but instead causes to poverty belt around the Capital, which is few around the world. Seen as a whole, Beijing, Tianjin and Hebei have no division of labor or collaboration at all inside, all are going on the way of its own. Hebei Province, as a big agricultural province, has more cooperation with Beijing and Tianjin in agriculture. However, in most cases, Hebei supplies agricultural products to Beijing and Tianjin, yet Beijing and Tianjin provides little agricultural technical services to Hebei, as a result, the cooperation is kept in a lower level.

The central government has repeatedly proposed the transfer of Beijing's traditional industries to Hebei, but achieved little due to the transfer relating taxes, even if Beijing will move large enterprises to Hebei, most of which are of high energy-consumption and the tax rights are still controlled by Beijing, and Hebei Province just achieves an increase in GDP; In addition, the low level industrial development and serious shortage of high-quality labor forces in Hebei Province also affects industrial connection to Beijing, instead, Tianjin, without sound industrial bases accepted the relocation of enterprises from Beijing, forming competition against Hebei Province. As for the tertiary industry, Beijing, Tianjin and Hebei all focus on the development, and the service industry in Beijing and Tianjin bring little pull to Hebei Province.

In a word, due to historical reasons as inequality in political status, vague orientation in urban function and so on, the collaborative development among Beijing, Tianjin and Hebei is not significant, instead, the hot competition is found between The situation will be improved gradually after the CPC Central Committee and State Council sets the collaborative development of Beijing, Tianjin and Hebei to a strategic height and defines the orientation between the three.

\section{KEY FIELD COOPERATION FOR BEIJING, TIANJIN AND HEBEI}

\section{A. Industrial Chain Division}

In the past 30 years after China's reform and opening up, urban economic circles have appeared such as the Pearl River Delta, the Yangtze River Delta, Beijing-Tianjin and Hebei region. Generally the economic development levels of all cities in a city circle differ, for example, Guangzhou, Shenzhen, Shanghai and Beijing own the highest development level in the circle, being the center of the economic circle, followed by Zhuhai, Suzhou and Tianjin, once again Jiangmen, Taizhou, Hebei. It is just the difference in the development level between cities that make them own different comparative advantages, e.g. the cities can achieve the work division in the different aspects of industrial chains, and avoid the industrial homogeneity for win-win. Traditional city circles as the Pearl River Delta, the Yangtze River Delta do well in this aspect, yet Beijing, Tianjin and Hebei fail to achieve dislocation development.

Previous studies show that Beijing, Tianjin and Hebei have serious homogenization in the leading industries, and few divisions and cooperation as between, but more in competition. Beijing, Tianjin and Hebei have different resource endowments, and functional orientation that the State endows also has characteristics of its own, Beijing, Tianjin and Hebei 
should strengthen deep-level division of labor and cooperation. However, after the collaborative development of Beijing, Tianjin and Hebei is upgraded to a national strategy, the phenomenon has changed. For example, Beijing takes the leading position in the development and utilization of new energy, and its new energy vehicle manufacturing industry has also reached a certain scale; Tianjin takes the leading in the manufacturing low-carbon environmental friendly equipment and battery production technology, and Hebei makes rapid progress in geothermal energy and solar energy.

Seen from the industrial chain, Beijing is at the top of the Beijing-Tianjin-Hebei region, strong in R\&D and innovation, but in lack of natural resources and limited environmental space. Tianjin is in the middle of the industrial chain, strong in industrial base and leading in manufacturing industry, the newly rising industries have reached a certain scale, yet the labor cost and land cost are relatively higher; Hebei Province is at the bottom of the industrial chain, general in the industrial base, but lower in labor cost and land cost, and rich in natural resources.

Therefore, Beijing can serve as a low-carbon technology R\&D center and the headquarters of low-carbon industry; Tianjin is the R\&D and transformation base to industrialize and market the low-carbon scientific research achievements; and Hebei Province, relying on advantages in costs, is the low carbon product base. As a result, the whole industrial chain of low-carbon economy can go well without obstacles, Beijing, Tianjin and Hebei perform duties of their own, sharing revenues, and the regional competitiveness will be greatly enhanced. In addition, special attention shall be paid that Hebei Province shall select to accept the industries from Beijing, but not devour. The task to govern environmental pollution is very heavy Hebei Province, it shall accept electronic information industry as possible as it can, but less high energy-consuming enterprises as metallurgy, chemical and so on, if having to accept, a low-carbon technical reform shall be conducted upon them.

\section{B. New Energy Field}

With the continuous development of China's economy, the energy consumption is also increasing, traditional energy not only brings higher carbon emission but also has its reserves declined, and thus, it is necessary to accelerate the development and utilization of new energy to change the status quo where the energy structure in China heavily depends on coal. Within the new energy sources that can be exploited currently in the natural world, solar energy is the best alternative to conventional energy sources, for the solar energy is inexhaustible, which requires neither development nor transportation, and brings no pollution to the environment. So the solar energy in recent years has become an important power source in most developed countries; In addition, wind energy is also an inexhaustible, renewable, non-polluting new energy. The Beijing-Tianjin-Hebei region has rich solar energy and wind energy. Especially the sunshine duration is longer in Baoding, Xingtai, Chengde, Zhangjiakou and so on, and the solar energy can be developed and used in a large scale; Zhangjiakou, Chengde, Qinhuangdao and Taihang Mountains have prominent advantages in wind resources.
Based on the relative advantages in new energy, Beijing, Tianjin and Hebei gradually define the labor division and form industrial chain with regional features. Strong in R\&D, Beijing aims at technical innovation of solar energy and wind energy; Gathering a large number of world-class new energy enterprises, Tianjin gradually builds itself into a R\&D results transformation base, devoting to industrialize and market new energy technologies; rich in solar energy and wind energy, Hebei has established large PV manufacturing base and new energy equipment production base, devoting to the large-scale and intensive production of light energy and wind energy, etc.

\section{Low Carbon Tourism Field}

The concept of low-carbon tourism was firstly presented at the 2009 World Economic Forum's "Low-Carbon Travel and Tourism" report. Low-carbon tourism, as the name implies, means that measures, as technical transformation at tourist attractions, rational design of travel routes and improvement of tourists' quality, will be taken to reduce the carbon emission in tourism, reduce the damages of tourism to bio-environment and achieve the low-carbon, green and environment-friendly travel. Compared with other traditional high-carbon industries, the carbon emission of tourism is not high, but the low carbon emission does not mean no carbon emissions, besides, Beijing, Tianjin and Hebei will take the tourism industry as a key industry in the future, so it will be of great significance to try to further reduce the carbon emission of tourism for the development of low-carbon economy development in Beijing, Tianjin and Hebei.

Both Beijing and Tianjin have a long history, thick in cultural atmosphere, and most tourism landscapes are historical and cultural relics; Hebei not only has places of interest as the Great Wall, the Summer Resort, but also lush forests, endless grassland, majestic mountains, beautiful coasts, vast lakes, beautiful scenery, etc. rich in tourism resources. The mutual supplement of tourism resources in Beijing, Tianjin and Hebei, together with the advantages in adjacent location and convenient transportation all have become good basis for the cooperation in tourism in Beijing, Tianjin and Hebei. Therefore, to strengthen the cooperation of Beijing, Tianjin and Hebei in tourism is an important opportunity for the development of low-carbon economy.

The region of Hebei Province surrounding the Capital has higher forest coverage, and there stand unique mounts and steep peaks, the vast grassland and fresh air, all are natural low-carbon tourism resources, suitable for the development of low-carbon tourism. Magnolia paddock, Yesanpo, Beidaihe and Gu'an Spring are all well-known leisure and tourism sites. Residents in Beijing and Tianjin with high income, have strong sense of environmental protection, and high demand for weekend excursions, Hebei Province shall catch the historic opportunity, give full priority to the advantages of excellent tourism resources and location of its own, and create a lowcarbon tourism brand.

Though the tourism resources of Beijing, Tianjin and Hebei are distinctive and complementary, yet if they are still fragmented, it will be not only a waste of resources but also fail to exert the synergistic effect of 1 plus 1 equals more than 
2. Therefore, Beijing, Tianjin and Hebei should break the division of administrative region, establish a unified lowcarbon tourism collaborative development agency, make an effective integration of three tourism resources, rational division, and form the most reasonable tourist routes according to different needs of tourists. Through the development of lowcarbon tourism, the Beijing-Tianjin-Hebei will be built into a leading low-carbon tourism demonstration region.

\section{Low Carbon Agriculture Field}

During the agricultural production, a large-scale use of pesticides and chemical fertilizers may in a short term bring huge gains, but have to make a higher payment in the long run. Therefore, traditional agriculture is a high-carbon industry, in order to develop the low-carbon economy, Beijing, Tianjin and Hebei must change the pattern of agricultural production to achieve low-carbon agriculture. The cooperation of Beijing, Tianjin and Hebei in the low-carbon agriculture may give more consideration to the following:

1) Make full effort for developing green and organic agriculture: Green and organic agriculture requires less water, chemical fertilizers and pesticides in the agricultural production and encourages the use of harmless fertilizers as bio-fertilizer and organic fertilizer so as to reduce the energy consumption and maintain the ecological balance. Beijing and Tianjin have higher mechanization and industrialization in agriculture, owning obvious advantages in capital and technology but narrow in geographical space, and the worst disadvantage is poor natural environment; though Hebei is poor in agricultural production technology and lower in mechanization and industrialization, yet the natural conditions are very favorable, and the open terrain, fertile soil, as well as certain agricultural base, makes is applicable for the largescale development of low-carbon agriculture.

In addition, there is large population in Beijing, Tianjin and Hebei, where the residents have higher income, having higher demand for healthy food, and the huge market demand will strongly drive the development of green and organic agriculture. Beijing and Tianjin, responsible for R\&D of lowcarbon agricultural technology, will provide Hebei Province with technical services and financing support; Hebei Province, responsible for the large-scale development of green and organic agriculture, will provide Beijing and Tianjin with healthy and marketable agricultural products.

2) Strengthen eco-protection: Beijing and Tianjin are much poor in eco-environment: lack of energy, scarce of water resources, lower vegetation coverage and sand blowing. In order to meet the demand of Beijing and Tianjin for energy and water and protect the eco-environment there, Hebei Province has made great sacrifices, in particular, the Zhangcheng area has its agricultural development strictly restricted in order to build a forest and sand shelter belt to safeguard Beijing and Tianjin. Suspension of heavy and chemical industry and restriction of agricultural development all make the region around Beijing and Tianjin become a poverty zone.

Therefore, Beijing, Tianjin and Hebei should establish an eco-compensation mechanism as between, it shall not only offer appropriate funds to compensate Hebei Province, but also provide policy supports, technical instruction, and build the poverty belt around the Capital into an eco-agricultural demonstration belt, enhance the region's self development function, expand the income sources of rural population. However, the establishment of eco-compensation mechanism will be involved in multiple interests, due to the existence of the administrative division, it requires the State to implement the top layout upon the mechanism.

3) Strengthen low-carbon technical training on farmers and instruct low-carbon planting: There stand colleges and universities in Beijing and Tianjin such as China Agricultural University, China Forestry University and so on leading in agriculture, there are the most excellent agricultural $R \& D$ talents here, owning the most advanced low-carbon agricultural technologies at home; Hebei Province should make good use of the advantage of close to Beijing and Tianjin, and invite agricultural technical experts to train and educate farmers, and teach farmers modern agricultural production modes, educate farmers in water saving, less fertilizer and pesticides.

\section{CONCLUSION}

Currently Beijing, Tianjin and Hebei have formed a consensus in the coordinated development: in order to solve the problem "big city disease" in Beijing, we must complete the transfer of the Capital's function; on the one hand, Tianjin shall strengthen its economic center, on the other hand, it should transfer some functions of its own; For Hebei, it, having no the effect of the Beijing-Tianjin region for years, becomes urgent to undertake the industrial transfer from Beijing and Tianjin to achieve a leapfrog development and shorten the economic and social gaps with that of Beijing and Tianjin.

\section{REFERENCES}

[1] Wen Kui, Zhu Erjuan, Beijing-Tianjin-Hebei Blue Book, BeijingTianjin-Hebei Integration Development Report (2012) [M] Beijing: Social Sciences Academic Press, Feb 2012

[2] Hao Jie, Beijing-Tianjin-Hebei Integration: Collaborative Development, Mutual Benefits and Win-Win [N] China Economic Information, Aug 14,2014

[3] Xi Jinping: Define Three-Place Functional Orientation to Achieve the Beijing-Tianjin-Hebei Integration Development [N] China Securities Journal, Feb 28, 2014

[4] Lu Xiaocheng, Drive the Beijing-Tianjin-Hebei Collaborative Development [J], Front, 2015(2) 